\title{
Natural Killer Cell Activity in Depressive Illness: Preliminary Report
}

\author{
Ziad Kronfol, Madhavan Nair, Joann Goodson, Kavita Goel, \\ Roger Haskett, and Stanley Schwartz
}

\section{Introduction}

The central nervous system and the immune system are closely interrelated. Stress and psychiatric illness have previously been associated with immune dysregulation (Calabrese et al. 1987). We reported earlier that patients with major depression manifested a reduction in mitogeninduced lymphocyte proliferation, a correlate of cellular immune function (Kronfol et al. 1983). These findings were later confirmed by other investigators (Schleifer et al. 1984). We now report our preliminary results on Natural-Killer (NK) cell activity, a more specific parameter of immune function directed against tumor- or virus-infected cells, in 12 drug-free patients diagnosed with major depressive disorder and 12 age- and sex-matched normal controls.

\section{Subjects and Methods}

\section{Subjects}

There werc 12 depressed paticnts, 5 mcn, 7 women (mean \pm SD age $36.8 \pm 9.3$ ). All patients were evaluated at the Department of Psy-

\footnotetext{
From the Departments of Psychiatry (Z.K., M.N., J.G., K.G., R.H.), Pediatrics (M.N., S.S.), and Epidemiology (M.N., S.S.), University of Michigan Medical Center, Ann Arbor, MI.

Supported in part by NIMH Grant MH-42988 and NIAAA Grant AA-07378.

Address reprint requests to Ziad Kronfol, M.D., Department of Psychiatry, University of Michigan Medical Center. UH-9C, Box 0120. 1500 E. Medical Center Drive, Ann Arbor, MI 48109. 0120

Received December 12, 1988; revised February 9. 1989.
}

chiatry of the University of Michigan Medical Center. Diagnosis was made independently by at least two psychiatrists in an unstructured interview using DSM-III criteria as well as Research Diagnostic Criteria (RDC) (Spitzer et al. 1977). The RDC diagnosis was confirmed by a structured interview conducted by a trained research nurse using the Schedule for Affective Disorders and Schizophrenia (SADS) (Spitzer and Endicott 1975). At the time of the study, 10 patients were hospitalized and 2 were ambulatory. They all met DSM-III and RDC criteria for major depressive disorder and had a score of $\geqslant 15$ on the 17-item Hamilton Depression Rating Scale, HDRS (Hamilton 1960). All patients were also free of drugs known to interfere with immune regulation, and free of psychoactive drugs for at least 2 weeks prior to immune evaluation. In fact, only 3 patients were receiving psychotropic medications (tricyclic antidepressants and/or neuroleptics) 2 weeks prior to the immunological assessment. The mean \pm SD score on the HDRS was $19.5 \pm 4.8$. The mean $\pm \mathrm{SD}$ score on the Carroll Depression Rating Scale (CDRS), a self-rating scale for depression (Carroll et al. 1981) was $29.0 \pm$ 9.1 .

The normal control group consisted of 12 healthy volunteers, 5 men, 7 women (mean \pm SD age $36.5 \pm 9.1)$, who were age and sex matched with the depressed patients. They were recruited in response to ads on the campus of the University of Michigan. Each control subject was interviewed with the SADS-Life Ver- 
sion (SADS-L), and subjects with a history of psychiatric illness were excluded. Other exclusion criteria were history of medical illness or drugs known to affect the immune system. The mean HDRS score was $0.6 \pm 0.9$. The mean CDRS was $2.1 \pm 2.5$.

Comparisons between the depressed patients and the normal controls revealed no significant age or sex differences between the groups. There were, however, highly significant differences in both the HDRS $(t=15.8 ; p<0.00001)$ and the CDRS $(t=7.6 ; p<0.0001)$ scores between the two groups.

\section{Methods}

Blood samples from each patient and his/her age-and-sex-matched normal control were drawn separately in heparinized tubes on the same day between 8:00 and 9:00 AM. Blood was diluted with phosphate-buffered saline (PBS pH 7.3) and centrifuged at $400 \mathrm{~g}$ for $30 \mathrm{~min}$ over a cushion of Ficoll-Hypaque solution. The mononuclear cell band was harvested, washed, and resuspended in RPMI 1640 medium containing 25 mM Hepes buffer supplemented with $10 \%$ heatinactivated fetal calf serum (Gibco, Grand Island, NY), $80 \mu \mathrm{g} / \mathrm{ml}$ gentamicin (Scherring Corp., Kenilworth, NJ), and $300 \mu \mathrm{g}$ of fresh glutaminc/ml (completc medium). Mononuclear cells were depleted of adherent/monocytes by chromatography on a column of Sephadex G10 beads (Pharmacia Fine Chemicals, Piscataway, N.J.) equilibrated in the same medium. The cell recovery was $>70 \%$ of the total input, and monocyte contamination as indicated by nonspecific esterase staining was $<2 \%$. NK activity was then measured in a direct ${ }^{51} \mathrm{Cr}$ release assay as described previously (Nair and Schwartz 1984). The human erythroleukemia cell line K562 was used as targets for NK cells. Four different effector:target (E:T) cell ratios were used: 50:1, 25:1: 12.5:1, and 6.25:1. All samples were run in triplicate. Percent cytotoxicity \pm SD was then calculated for both patients and controls for each of the four different E:T cell ratios. The spontaneous release for labeled K562 target cells was always less than $10 \%$ of the total labeling.

\section{Statistical Analysis}

Differences between patients and their matched controls were calculated for each E:T cell ratio using a paired $t$-test analysis. Differences between patients and controls were also analyzed using repeated measures analysis of variance (ANOVA). Results were considered statistically significant wherever $p<0.05$, two-tailed.

\section{Results}

Figure 1 shows mean \pm SD percent cytotoxicity of lymphocytes from depressed patients and their matched controls against K562 target cells. Depressed patients had lower percent cytotoxicity values than their matched controls for all $\mathrm{E}: \mathrm{T}$ cell ratios used. These differences were statistically significant at E:T cell ratio 50:1 $(t=$ $2.97, \mathrm{n}=12, p<0.02$ ) and $\mathrm{E}: \mathrm{T}$ cell ratio $25: 1$ $(t=3.1, \mathrm{n}=12, p<0.02)$. A decreased cytotoxicity was also demonstrated at the lowest E:T cell ratios 12.5:1 $(t=1.89 \mathrm{n}=12 p<$ $0.09)$ and 6.25:1 $(t=0.91 \mathrm{n}=12$; NS $)$, although these values were not significantly different from controls.

To assess the overall difference between the two groups, we then used repeated measures ANOVA of percent cytotoxicity between the groups. We found a significant group effect ( $F$ $=4.92 ; \mathrm{df}=1 ; p<0.05)$, a significant dose effect $(F=247.31$; df $=3 ; p<0.0001)$ and a significant group by dose interaction $(F=$ 5.33; $\mathrm{df}=3 ; p<0.01)$.

\section{Discussion}

These results suggest that NK cell activity, a kcy component of the immune response, is decreased in patients suffering from a major depressive episode. NK activity, like other defense mechanisms, can be affected by several clinical factors, including age, gender, nutritional status, diurnal variation, medical illness, and drugs. Patients and controls did not significantly differ in either age or gender distribution. Similarly, there were no significant differences in thcir respective mean body weights $(73.0 \pm 18.5 \mathrm{~kg}$ for depressed patients, $65.3 \pm 10.9 \mathrm{~kg}$ for nor- 


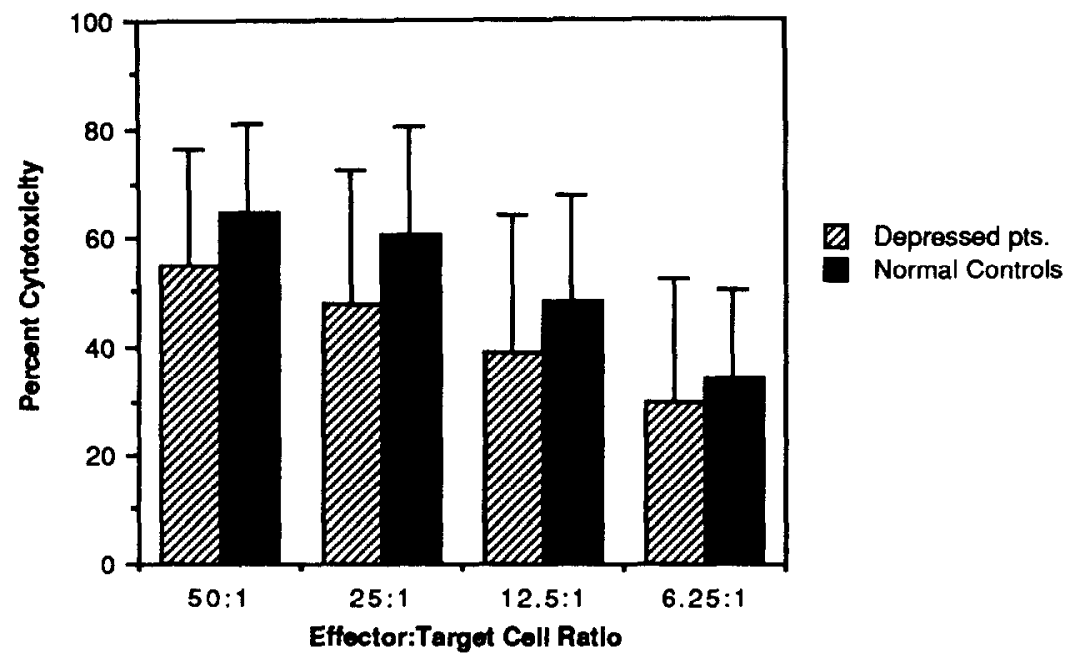

Figure 1. NK activity in depressed patients and matched normal controls. The results are expressed as mean \pm SD percent cytotoxicity of lymphocytes from 12 depressed patients and 12 matched controls. All samples were run in triplicate for each of four different E:T cell ratios. The difference between the two groups was statistically significant $(p<0.05$, ANOVA).

mal controls; $t=1.6$; NS). All blood samples were drawn at the same time of the day, and assays were run simultaneously on fresh blood samples from depressed patients and their matched controls. Patients were excluded if they met criteria for psychiatric disorders other than major depression. Normal controls had no evidence of psychiatric illness. Both patients and controls were physically healthy and free of drugs known to affect immune regulation, including psychoactive drugs.

A reduction in NK activity may represent an important feature of immunoregulation in patients with depressive illness. It should, however, be noted that this finding is not universally seen in patients with major depression. In the present study, only 7 of the 12 patients under investigation had significantly lower NK activity (by paired $t$-test analysis) than their matched controls. The other 5 patients did not differ significantly from their matched controls. In no instance did any depressed patient show a significantly higher NK activity than his/her matched control. Clinical and neuroendocrine factors that distinguish depressed patients with impaired NK activity from those with normal NK activity are presently under investigation. More work is needed to clarify the relation among clinical, neuroendocrine, and immune phenomena in patients with depressive illness.

\section{References}

Calabrese J, Kling M, Gold PW (1987): Alterations in immunocompetence during stress, bereavement and depression: Focuses on neuroendocrine regulation. Am I Psvchiatry 144:1123-1134

Carroll BJ, Feinberg M, Smouse P, Rawson S, Greden JF (1981): The Carroll Rating Scale for Depression. 1. Development, Reliability and Val idation. Br J Psychiatry 138:194-200.

Hamilton M (1960): A rating scale for depression. $J$ Neurol, Neurosurg Psychiatry 23:56-62.

Kronfol Z, Silva J, Greden J, Dembinski S, Gardner R, Carroll B. (1983): Impaired lymphocyte function in depressive illness. Life Sci 33:241247.

Nair MPN, Schwartz SA (1984): Immunomodulatory effects of corticosteroids on natural killer and antibody-dependent cellular cytotoxic activities on human lymphocytes. I Immunol 132:28762882.

Schleifer SJ, Keller SE, Meyerson AT, Raskin MJ, Davis KL, Stein M (1984): Lymphocyte function 
in major depressive disorder. Arch Gen Psychiatry 148:70-73.

Spitzer R. Endicott J (1975): Schedule for Affective Disorders and Schizophrenia. New York: New York State Psychiatric Institute.
Spitzer R, Endicott J, Robins E (1977): Research Diagnostic Criteria $(R D C)$ for a Selected Group of Functional Disorders. New York: New York State Psychiatric Institute.

\title{
Ventricle-to-Brain Ratio in Schizophrenia: A Controlled Follow-up Study
}

\author{
Dargut Kemali, Mario Maj, Silvana Galderisi, Nicola Milici, \\ and Antonio Salvati
}

\section{Introduction}

The presence of enlarged lateral cerebral ventricles on computed tomography (CT) scans of a subset of schizophrenic patients has been reported by several authors (for a review, see Shelton and Weinberger 1986). This enlargement is commonly believed to predate the onset of illness and to be nonprogressive: in fact, most studies found no correlation between lateral ventricle size and duration of illness, and ventricular enlargement was also observed in young patients with schizophreniform disorder (Weinberger et al. 1982). Nevertheless, controversy on this topic persists. It has been argued (Woods and Wolf 1983) that the relationship between ventricle size and duration of illness was missed in some investigations as a consequence of the homogeneity of the patient sample with respect to the duration itself. Indeed, some studies ex-

\footnotetext{
From the Department of Medical Psychology and Psychiatry, First Medical School. University of Napies, Italy.

Address reprint requests to Prof. Dargut Kemiali, Clinica Psichiatrica. Primo Policlinico Universitario, Piazza Miraglia 2, 1-80138 Napoli. Italy.

Received September 2, 1988 ; revised February 3. 1989.
}

amining patient samples that were highly heterogeneous with respect to duration of illness reported a significant correlation between such a variable and the ventricle-to-brain ratio (Kemali et al. 1987).

The question of whether the enlargement of lateral ventricles in schizophrenia antedates the onset of illness or occurs during its course can be clarified only by longitudinal studies. However, only three investigations of this type have been published until now (Nasrallah et al. 1986; Illowsky et al. 1988; Vita et al. 1988), of which the first was affected by important methodological problems (follow-up only of patients who continued to attend the unit as outpatients, use of different CT scanners at baseline and at follow-up), and none included a control population. This is the reason why the present controlled follow-up study was carried out.

\section{Methods}

In a previous study (Kemali et al. 1987), we performed CT scan in 50 patients with a DSMIII diagnosis of schizophrenic disorder and no history of alcoholism and drug abuse, who had 